\section{Arthroscopic Coblation Saucerisation of Discoid Medial Meniscus - A Novel Technique}

Edmund leong*, Piyush Mahapatra and Christopher P Huber

West Middlesex University Hospital, Isleworth, Middlesex, United Kingdom

\begin{abstract}
Background: Discoid medial meniscus is a rare clinical presentation, and the prevalence of lateral discoid meniscus is higher and has been more widely reported. The recommended treatment of symptomatic discoid meniscus is partial excision and saucerisation.

Case description: We report on a 29 year old male with a symptomatic medial discoid meniscus, who underwent arthroscopic saucerisation using an arthroscopic coblation technique.

Outcomes: At 5 month follow up, the patient's symptoms of pain had completely resolved. He reported better everyday functional activities and demonstrated an improvement in knee range of motion.

Discussion: There have been many reports of discoid meniscus saucerisation using a variety of techniques. To our knowledge, there have been no reports of arthroscopic coblation for a symptomatic discoid meniscus. We describe the advantages to our technique, with reference to current literature on discoid meniscus.

Keywords: Arthroscopy; Discoid meniscus; Menisectomy; Saucerisation
\end{abstract}

\section{Introduction}

\section{Background}

The first account of a discoid meniscus was reported by Young [1] in 1889, when he reported two cases of lateral discoid menisci. Since then, there have been multiple reports of lateral discoid menisci [2-6]. In 1930, Watson-Jones [7] reported an anomaly of the medial meniscus when he treated a much less common medial "ring" meniscus in a 34-year-old man. The medial discoid meniscus was then reported in two separate patients by Cave and Staples [8] in 1941. In 1956, Murdoch [9] reported the first case of bilateral medial discoid

*Corresponding author: Edmund leong, West Middlesex University Hospital, Twickenham Rd, Isleworth, Middlesex, TW7 6AF, United Kingdom, Tel: +44 2085602121; E-mail: edieongortho@gmail.com

Citation: leong E, Mahapatra P, Huber CP (2015) Arthroscopic Coblation Saucerisation of Discoid Medial Meniscus - A Novel Technique. J Orthop Res Physiother 1: 017

Received: September 22, 2015; Accepted: November 12, 2015; Published: December 01, 2015 menisci in a 43-year-old man. This patient experienced typical clicking with pain in both knees. The patient did well postoperatively after both medial menisci were excised in separate operations.

The discoid medial meniscus is rare with an estimated prevalence of $0.12 \%$ [10]. The prevalence of a lateral discoid meniscus is higher and has been reported to $1.5 \%$ [10]. The clinical signs and symptoms of discoid medial meniscal injuries are similar to those of any other meniscal injury. Case reports and case series have recommended partial excision, rather than total menisectomy $[6,9,10]$.

Coblation is advanced technology that quickly and effectively removes and shrinks soft tissue using radio-frequency energy. It is not a heat-driven process, and therefore surrounding healthy tissue is preserved. We describe a simple technique for saucerisation by using coblation with the Arthocare Super MultiVac 50 Arthrowand, in a rare case of a symptomatic discoid meniscus.

\section{Case Description}

The patient is a 29 year old male who was referred to the physical therapist with a 3-4 year history of right medial knee pain, worse when standing for prolonged periods of time. He described intermittent swelling of the knee, with difficulty ascending and descending stairs. He also reported an inability to stand on the right leg alone, and an inability to fully flex and extend his knee. There was no previous history of any significant trauma to the knee. He did not complain of any locking nor clicking, or any symptoms of instability. He has no significant past medical history of note and has not had any previous surgeries. He worked as a waiter, but recently had to give up his job due to worsening knee symptoms. He has always found playing sports difficult due to his knee symptoms, particularly football. On examination, he had a mild right sided antalgic gait and a moderate knee joint effusion. His knee range of motion was 10 degrees fixed flexion to 120 degrees. He had discrete medial sided joint line tenderness. McMurray's test was positive for pain. There were no clinical signs of ligamentous instability of the knee.

Plain weight bearing radiographs of the knee were initially reported as normal by the radiologist. In retrospect, following Magnetic Resonance Imaging (MRI), the radiographs had actually demonstrated subtle radiological signs of a discoid meniscus; a widened joint space, squaring of the condyles and spur formation (Figure 1).

He was initially managed non-operatively, for 6 months, with activity modification, physiotherapy, and non steroidal anti-inflammatory medication. He did not experience any relief or improvement in his symptoms. Due to lack of progress a MRI scan was performed, which demonstrated a complete medial discoid meniscus (Figure 2).

He was therefore referred to the orthopaedic surgeon. Our patient's long history of significantly troubling knee symptoms had a detrimental effect on his daily activities and working life. In view of this, and his failure to progress and lack of benefit from non-operative management, he was appropriately counselled and consented for arthroscopic saucerisation of the discoid meniscus, followed by physical therapy. 


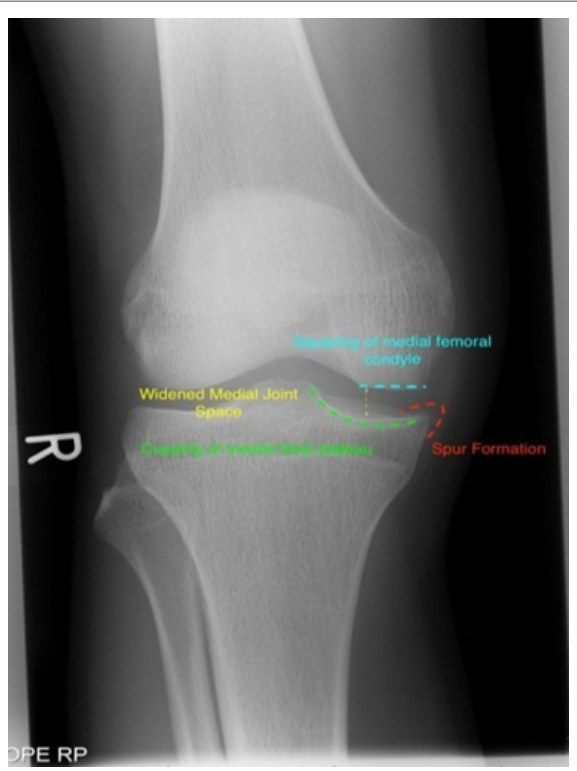

Figure 1: Radiological features of a discoid meniscus - a widened joint space, squaring of the condyles and spur formation.

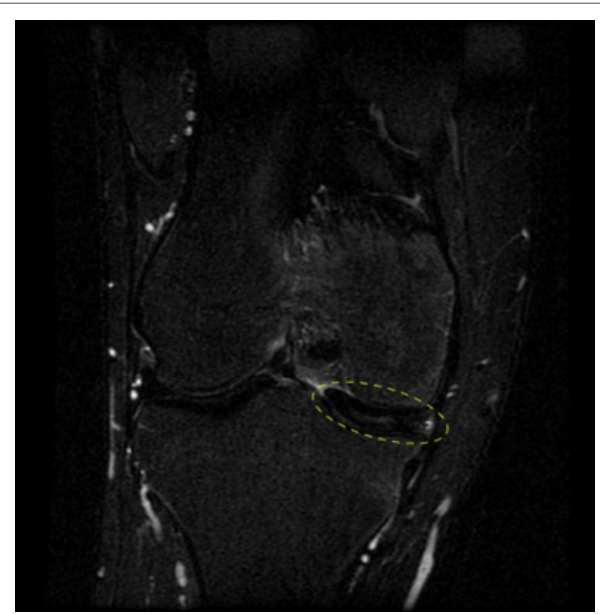

Figure 2a: Magnetic resonance image of discoid meniscus (Coronal T2 weighted STIR sequence).

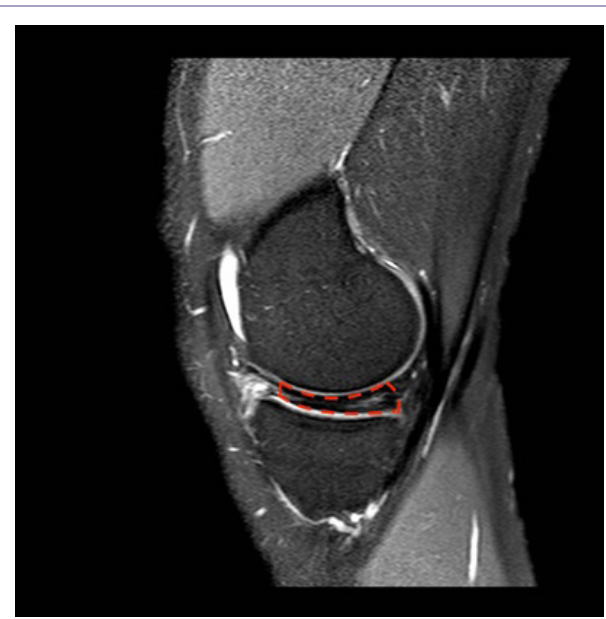

Figure 2b: Magnetic resonance image of discoid meniscus (Sagittal T2 PDW SPAIR proton density weighted sequence)

\section{Surgical Technique}

Standard anterolateral and anteromedial portals are placed slightly higher to avoid the anterior portion of the discoid meniscus. These are located approximately $0.5 \mathrm{~cm}$ lateral to the inferior edge of the patella. The high anteromedial portal position is determined with direct visualisation of a localising needle. This allows accurate evaluation of the working dimensions of the portal and minimises the risk of chondral damage. First the discoid meniscus is examined for any tears or other abnormalities (Figure 3). Then the surgeon must evaluate the edges and margins to be excised. In this case, the discoid meniscus was complete with no discrete tears. The Arthrocare Arthrowand Super Multi-Vac 50 is then introduced and the coblation of the meniscus begins in the mid portion. Once the appropriate depth is reached (in our case the full depth of the meniscus), the rest of the coblation continues outwards to the predetermined margins of resection, whilst taking care to avoid damage to the tibial chondral surface (Figure 4 and 5). One must be careful to avoid iatrogenic chondral damage by meticulous use of the coblator and use frequent and short bursts of the coblator to avoid iatrogenic chondral damage and to keep the temperature of the probe and environment to a minimum. Any edges may be trimmed using the arthroscopic shaver or the Arthrowand as necessary. The remaining meniscus is evaluated for adequacy of resection, stability, and degenerative tears (Figure 6).
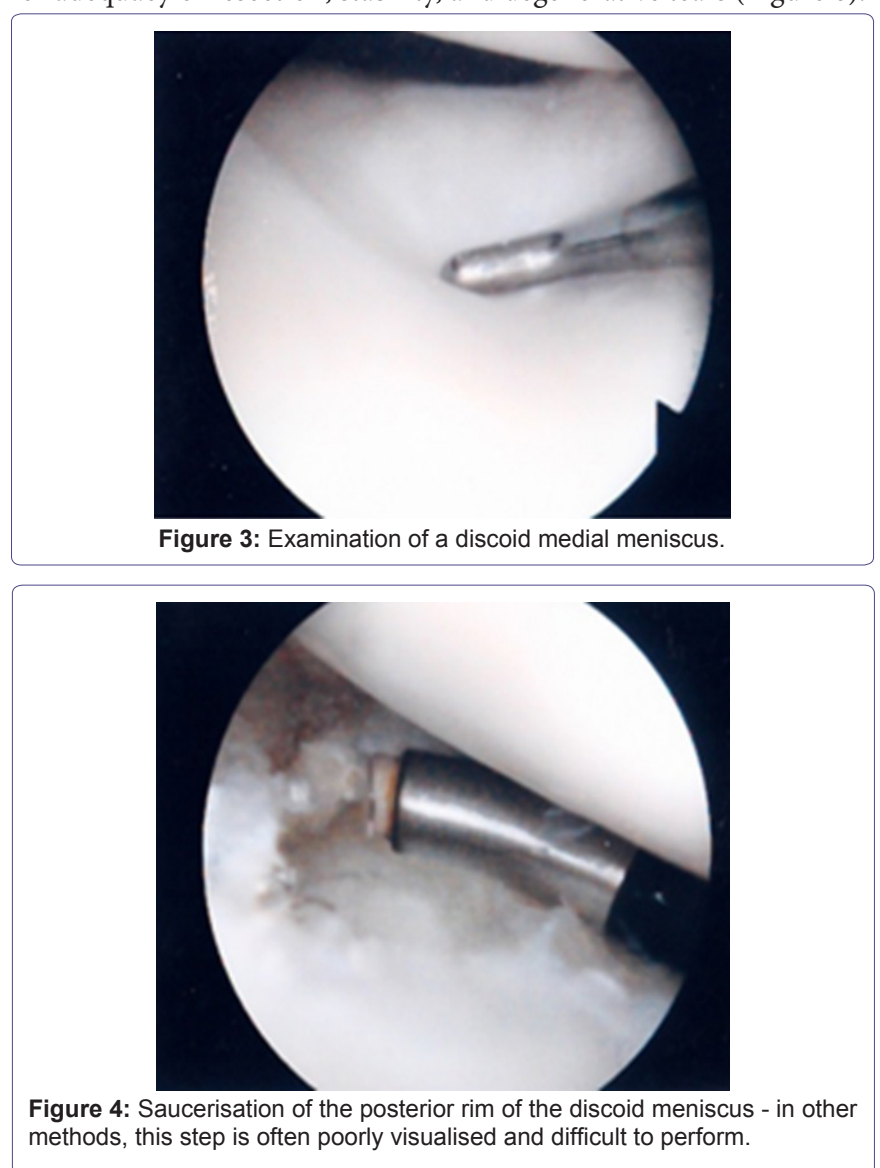

\section{Outcomes}

At six weeks follow up in the clinic, the patient reported an $80 \%$ improvement in pain symptoms, according to a pain Visual Analogue Scale (VAS). At the 6 month follow up, his medial sided knee pains had completely resolved. He also reported improvements in function; the procedure had resolved the medial sided pains when he drives his 


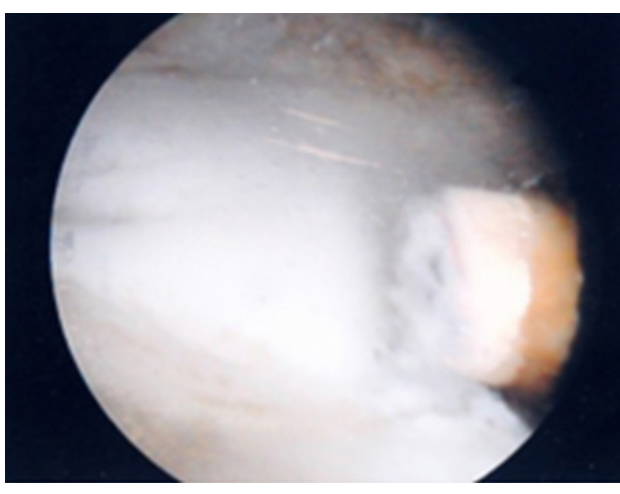

Figure 5: The anterior rim of the discoid meniscus is easily coblated with the 50 degree Arthrowand.

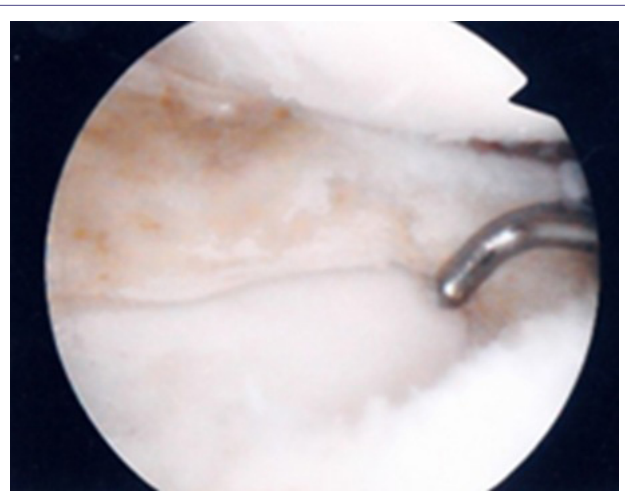

Figure 6: Successful saucerisation of a medial discoid meniscus with preservation of chondral surfaces.

car and he is now able to stand unsupported on the operated side. He has had no mechanical symptoms of clicking, locking and no episodes of giving way. On examination, he is now able to extend to 5 degrees and flex to 145 degrees, with a negative McMurray's test to pain. He is currently under long term follow up.

\section{Discussion}

The discoid medial meniscus is rare with an estimated prevalence of $0.12 \%$ [10]. Surgical intervention is often required for symptomatic discoid meniscus. Biomechanical studies have shown that the meniscus plays an important function in load transmission, stability, lubrication and nutrition of the articular cartilage $[6,7,11,12]$. Historically, total menisectomy was the treatment of a symptomatic discoid meniscus, however this results in progressive osteoarthritis and a poor prognosis [13]. Arthroscopic partial excision and/or saucerisation of the meniscus have been recommended for symptomatic discoid meniscus and better results have been reported with this $[6,9,14]$. Ogata [6] demonstrated excellent results in $86 \%$ of 76 patients who underwent partial menisectomy for symptomatic discoid meniscus, with no patients requiring repeat operations for poor results.

The meniscus in the normal knee joint plays an important function, thus partial menisectomy is the preferred arthroscopic treatment of symptomatic discoid meniscus $[6,14,15]$. There have been many reported methods of arthroscopic partial menisectomy such as open excision, one or two piece excision, and piece-meal arthroscopic excision [3,16-18]. Piecemeal excision of a discoid meniscus can potentially be difficult to perform if working space is limited, including the need for a larger portal to remove a large piece of meniscus [18].
It is important to leave a rim of meniscus, free of any remaining tears. By doing so, the remaining portion performs the function of the meniscus, preventing instability from total menisectomy [3]. The width of the remaining meniscus rim are dependent on the type of meniscus and on the shape and extent of the torn meniscus [16]. Hayashi et al., [16] left widths of $6 \mathrm{~mm}$ and $8 \mathrm{~mm}$ for complete and incomplete type lesions respectively. Ogata [6] would routinely leave a peripheral rim of 6 to $8 \mathrm{~mm}$. Vandermeer and Cunningham [19] have left widths of 4 to $5 \mathrm{~mm}$. We felt a width of 5 to $6 \mathrm{~mm}$ provided a stable rim with no impingement of the femoral condyle against the remaining rim.

Arthroscopic treatment of a complete discoid meniscus is a technically demanding procedure. The one piece excision technique described by Kim [17] requires a third portal, repeated changes in instrumentation, and a large portal for removal of a large piece of meniscus. Other piecemeal excision techniques can prove to be difficult and time consuming, with difficulty in initiating the excision point, frequent passage of instruments and the possibility of leaving excised meniscus in the joint [18].

To our knowledge, there have been no reports of arthroscopic coblation for saucerisation of a discoid meniscus. We found that there were a number of advantages to our technique compared with other described techniques. We had few changes in instrumentation when resecting a portion of the discoid meniscus. The progressive nature of resection allows constant evaluation of the amount resected and the shape of the remaining rim, whereas many other techniques involve excising the portion in one go. It also avoids piecemeal excision, which would otherwise produce free floating pieces of meniscus which need to be removed. The Arthrowand suction draws free-floating tissue to the electrodes, negating the need for chasing evasive tissue. This has the added advantage of reduced operative tourniquet times. The 50 degree angle on the coblator allows a wider degree of freedom and working angle, which we found to be suitable for shaping the meniscus. Post-operative MR scans demonstrate the remaining rim of meniscus, closely resembling a 'normal shaped meniscus' and preserved chondral surfaces (Figure 7). This technique is theoretically reproducible with different manufactures of coblators. Our reasons for using the Arthrocare Arthrowand Super Vac 50 was due to surgeon training/familiarity and product availability as well as some of the features of this product. The estimated temperature at the tip of the instrument is 20-60 degrees centigrade. Safety features include a

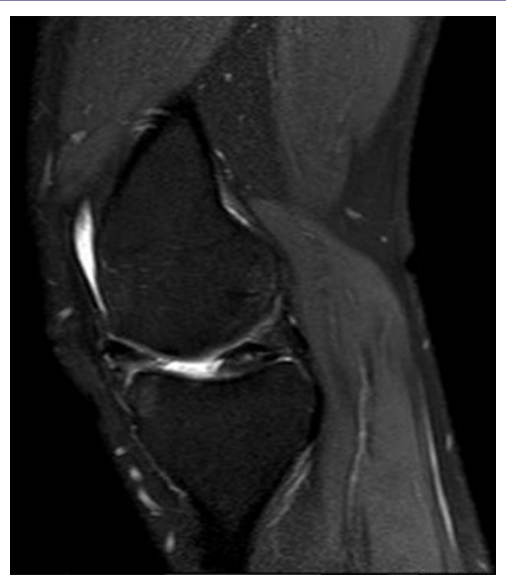

Figure 7: Post-operative MRI demonstrates the remaining rim of meniscus, closely resembling a 'normal shaped meniscus' and preserved chondral surfaces (Sagittal T2 weighted PDW SPAIR sequence). 
$100 \mu \mathrm{m}-200 \mu \mathrm{m}$ plasma field allows for precise removal of soft tissue with minimal damage to untargeted tissue. Nevertheless meticulous technique and care must still be taken to avoid iatrogenic damage. Various manufacturers offer different technologies to the safety and efficacy of their products, but it is not within the scope of this article to discuss further. Although this is presented as a new technique for a rare condition, we see no reason why this technique may not be applied to more common presentations, such as a lateral discoid meniscus or meniscal tears, but strongly advocate proper training and education in the use of such instruments. We also eagerly await the long terms results following our use of this technique to confidently confirm adequacy of resection and the benefits and safety of this technique.

In summary, we have demonstrated a simple technique for the saucerisation of a medial discoid meniscus which is easy and quick to perform in comparison with other known techniques.

\section{References}

1. Woods GW, Whelan JM (1990) Discoid meniscus. Clin Sports Med 9: 695706.

2. Berson BL, Hermann G (1979) Torn discoid menisci of the knee in adults. Four case reports. J Bone Joint Surg Am 61: 303-304.

3. Flouzat-Lachaniette $\mathrm{CH}$, Pujol N, Boisrenoult P, Beaufils P (2011) Discoid medial meniscus: report of four cases and literature review. Orthop Traumatol Surg Res 97: 826-832.

4. Hayashi LK, Yamaga H, Ida K, Miura T (1988) Arthroscopic meniscectomy for discoid lateral meniscus in children. J Bone Joint Surg Am 70: 1495-1500.

5. Ikeuchi $H$ (1982) Arthroscopic treatment of the discoid lateral meniscus. Technique and long-term results. Clin Orthop Relat Res: 19-28.

6. Ogata K (1997) Arthroscopic technique: two-piece excision of discoid meniscus. Arthroscopy 13: 666-670
7. Jones RW (1930) Specimen of internal semilunar cartilage as a complete disc. Proc R Soc Med 23: 1588-1589.

8. Cave EF, Staples OS (1941) Congenital discoid meniscus: A cause of internal derangement of the knee. The American Journal of Surgery 54: 371-376.

9. Kose O, Celiktas M, Egerci OF, Guler F, Ozyurek S, et al. (2015) Prognostic factors affecting the outcome of arthroscopic saucerization in discoid lateral meniscus: a retrospective analysis of 48 cases. Musculoskelet Surg 99: 165170.

10. Dickason JM, Del Pizzo W, Blazina ME, Fox JM, Friedman MJ, et al. (1982) A series of ten discoid medial menisci. Clin Orthop Relat Res: 75-79.

11. Baratz ME, Fu FH, Mengato R (1986) Meniscal tears: the effect of meniscectomy and of repair on intraarticular contact areas and stress in the human knee. A preliminary report. Am J Sports Med 14: 270-275

12. Walker PS, Erkman MJ (1975) The role of the menisci in force transmission across the knee. Clin Orthop Relat Res: 184-192.

13. Cox JS, Nye CE, Schaefer WW, Woodstein IJ (1975) The degenerative effects of partial and total resection of the medial meniscus in dogs' knees. Clin Orthop Relat Res: 178-183.

14. McGinity JB, Geuss LF, Marvin RA (1977) Partial or total meniscectomy: a comparative analysis. J Bone Joint Surg Am 59: 763-766.

15. Kim SJ, Yoo JH, Kim HK (1996) Arthroscopic one-piece excision technique for the treatment of symptomatic lateral discoid meniscus. Arthroscopy 12: 752-755.

16. Fujikawa K, Iseki F, Mikura Y (1981) Partial resection of the discoid meniscus in the child's knee. J Bone Joint Surg Br 63: 391-395.

17. Jeannopoulos CL (1950) Observations on discoid menisci. J Bone Joint Surg Am 32-A: 649-652.

18. Murdoch G (1956) Congenital discoid medial semilunar cartilage. J Bone Joint Surg Br 38-B: 564-566.

19. Vandermeer RD, Cunningham FK (1989) Arthroscopic treatment of the discoid lateral meniscus: results of long-term follow-up. Arthroscopy 5: 101-109. 